Annales Geophysicae (2002) 20: 57-67 C European Geophysical Society 2002

\title{
Excitation of Alfvén waves by modulated HF heating of the ionosphere, with application to FAST observations
}

\author{
E. Kolesnikova, T. R. Robinson, J. A. Davies, D. M. Wright, and M. Lester \\ Department of Physics and Astronomy, University of Leicester, Leicester LE1 7RH, UK
}

Received: 7 February 2001 - Revised: 18 June 2001 - Accepted: 19 June 2001

\begin{abstract}
During the operation of the EISCAT high power facility (heater) at Troms $\varnothing$, Norway, on 8 October 1998, the FAST spacecraft made electric field and particle observations in the inner magnetosphere at 0.39 Earth radii above the heated ionospheric region. Measurements of the direct current electric field clearly exhibit oscillations with a frequency close to the modulated frequency of heater $(\sim 3 \mathrm{~Hz})$ and an amplitude of $\sim 2-5 \mathrm{mV} \mathrm{m}^{-1}$. Thermal electron data from the electrostatic analyser show the modulation at the same frequency of the downward electron fluxes. During this period the EISCAT UHF incoherent scatter radar, sited also at Troms $\varnothing$, measured a significant enhancement of the electron density in E-layer up to $2 \cdot 10^{12} \mathrm{~m}^{-3}$. These observations have prompted us to make quantitative estimates of the expected pulsations in the inner magnetosphere caused by the modulated HF heating of lower ionosphere. Under the conditions of the strong electron precipitation in the ionosphere, which took place during the FAST observations, the primary current caused by the perturbation of the conductivity in the heated region is closed entirely by the parallel current which leaks into the magnetosphere. In such circumstances the conditions at the ionosphere-magnetosphere boundary are most favourable for the launching of an Alfvén wave: it is launched from the node in the gradient of the scalar potential which is proportional to the parallel current. The parallel electric field of the Alfvén wave is significant in the region where the electron inertial length is of order of the transverse wavelength of the Alfvén wave or larger and may effectively accelerate superthermal electrons downward into the ionosphere.
\end{abstract}

Key words. Ionosphere (active experiments; ionosphere magnetosphere interactions; particle acceleration)

\section{Introduction}

Observations reported in a recent paper by Robinson et al. (2000) clearly demonstrated that ULF waves observed by

Correspondence to: E. Kolesnikova (elka@ion.le.ac.uk) the FAST satellite at an altitude of $2550 \mathrm{~km}$ were caused by the modulated heating of the lower ionosphere by the EISCAT high power facility at Troms $\varnothing$, Norway. $3 \mathrm{~Hz}$ oscillations with an amplitude of about $2-5 \mathrm{mV} \mathrm{m}^{-1}$ were detected by the FAST electric field antenna during approximately 3 seconds when the satellite track passed above the heated patch of ionosphere. Robinson et al. (2000) also reported observations of $3 \mathrm{~Hz}$ oscillations in the downward directed component of electron fluxes which they attributed to parallel electric field caused by electron inertia effect associated with an Alfvén wave. A limited number of papers (see, for example, Kimura et al., 1994 and references in it) have reported heater induced VLF waves detected by spacecraftborne receivers when spacecraft passed in the vicinity of Troms $\varnothing$ heater. The above-mentioned FAST observations constitute a first space detection of artificial ULF waves.

A detailed theoretical analysis of artificial magnetic pulsations in the low frequency range, caused by modulated HF heating of the ionosphere has been carried out previously by Stubbe and Kopka (1977), Pashin et al. (1995), Lyatsky et al. (1996), Borisov and Stubbe (1997). The last authors included the field-aligned current and constructed a quantitative model of the generation of three-dimensional currents due to the periodic heating which produce both magnetic perturbations on the ground and also Alfvén waves propagating upward from the upper ionosphere. In our paper we follow the approach proposed by Borisov and Stubbe (1997) and develop it further for the conditions of strong electron precipitation, with the aim of determining the current system excited in the ionosphere by the modulated heating of the lower ionosphere.

We begin in Sect. 2 by briefly reviewing the heater experimental arrangements, geophysical conditions during the heater operation and related FAST observations. In Sect. 3, we give, (i) a discussion of the HF heater wave absorption and the resulting heating effect in the absorption layer, (ii) a brief overview of the model proposed by Borisov and Stubbe (1997) and its development for the case of strong skin effect and (iii) results of the model applied to the ionospheric conditions observed during the FAST measurements. 


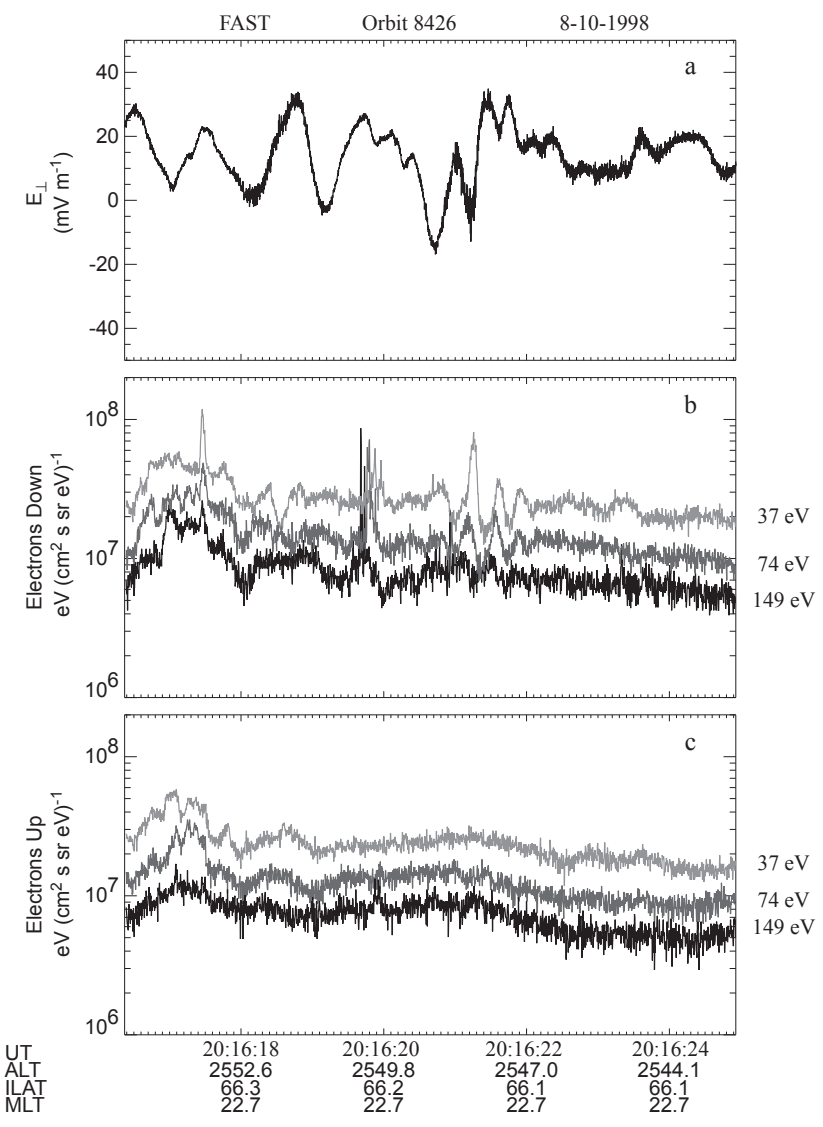

Fig. 1. The same as Fig. 2 in the paper by Robinson et al. (2000). FAST observations: (a) perpendicular component of dc electric field; (b) and (c) downward/upward electron fluxes in the 37, 74, $149 \mathrm{eV}$ channels of the electrostatic analyzer, presented in the units of fluxes multiplied by the central energy of the corresponding energetic channel.

In Sect. 4 we discuss properties of the Alfvén wave associated with a leakage of parallel current from the ionosphere and the acceleration of superthermal electrons by the parallel electric field of a modified Alfvén wave in which electron inertia has been explicitly included.

\section{Experiment and observations}

\subsection{Modulated HF heater radiation}

During the period of modulated heating, between 20:15 UT and 20:45 UT on 8 October 1998, the Troms $\varnothing$ heater transmitted $4.04 \mathrm{MHz}, X$-mode radio waves into a cone with a half-width of $7^{\circ}$, centered along the direction of the local magnetic field. The HF signal was modulated by the squarewave with a fundamental frequency of $3 \mathrm{~Hz}$ and effective radiated power $W_{o}$ of approximately $2 \cdot 10^{5} \mathrm{~kW}$. Hereafter, we assume that higher harmonics may be neglected, therefore the variation of the modulated HF electric field at a height $z$ and at a horizontal distance $r$ from the transmitter takes the form (Gurevich, 1978)

$E_{o}(r, z) \approx 300 \frac{\sqrt{W_{o} / 4}}{z} e^{-r^{2} / a^{2}}(1+\gamma \cos \Omega t)$,

where $E_{o}$ is given in $\mathrm{mV} \mathrm{m}^{-1}, W_{o}$ in $\mathrm{kW}, z$ in $\mathrm{km}, a$ is a radius of the heated spot ( $\sim 10 \mathrm{~km}$ at a height $80 \mathrm{~km}), \Omega$ is the fundamental modulated frequency $\left(\Omega=18.8\right.$ radians $\left.\mathrm{s}^{-1}\right)$ and $\gamma \approx 2 / \pi$.

\subsection{FAST observations}

As reported by Robinson et al. (2000), during the above experiment, the electric field antenna installed on the board the FAST satellite observed $3 \mathrm{~Hz}$ waves with an amplitude of $\sim 2-5 \mathrm{mV} \mathrm{m}^{-1}$ between $\sim 20: 16: 20 \mathrm{UT}$ and $\sim 20: 16: 23 \mathrm{UT}$ at an altitude of $2550 \mathrm{~km}$ (Fig. 1a). The electric field antenna was oriented approximately perpendicular to the magnetic field line. Simultaneous measurements of the electron fluxes in three energetic channels of the electrostatic analyser with the central energies: 37,74 and $149 \mathrm{eV}$ show a corresponding quasi-periodic acceleration of the downward electrons (Fig. 1b). Each energy band exhibits similar oscillations with a common period of $3 \mathrm{~Hz}$. However, they have phases different from that of the electric field oscillations and also different from each other. The fact that the phases of the more energetic electrons lead is consistent with a source of the electron fluxes at several hundred $\mathrm{km}$ above the observation point. Note that the upgoing electrons in the same energy bands (Fig. 1c) do not exhibit any significant $3 \mathrm{~Hz}$ modulation. A detailed discussion of the mechanism which causes the electron acceleration is given in Sect. 4 .

\subsection{Geophysical conditions}

The electron density profile at altitudes between 80 and $600 \mathrm{~km}$ above the heater patch was obtained from observations of the EISCAT UHF incoherent scatter radar located in the vicinity of the Troms $\varnothing$ heater. The density profile in Fig. 2a corresponds to the radar integration period of 10 seconds which commences at 20:16:20 UT and consequently covers the period of the FAST observations. A significant enhancement of the electron density in E-layer up to $2 \cdot 10^{12} \mathrm{~m}^{-3}$ was due to the increase precipitation of electrons into ionosphere during the experiment. The bulk velocity of the plasma at $250 \mathrm{~km}$ above the Troms $\varnothing$ heater, as estimated using tristatic measurements from the three UHF receivers during a $\sim 30 \mathrm{~s}$ timeperiod, had the main component directed towards north with an amplitude of $\sim 240 \mathrm{~m} \mathrm{~s}^{-1}$. Consequently the electric field associated with the $\boldsymbol{V} \times \boldsymbol{B}_{o}$ drift pointed eastwards, with an amplitude of $\sim 12 \mathrm{mV} \mathrm{m}^{-1}$. Molecular composition and temperature in the midnight sector as deduced from the ionospheric model MSIS90 (Hedin, 1991) are presented in the Figs. $2 b$ and c. The electron temperature is taken as that for the neutral gas at the low altitudes, $z<250 \mathrm{~km}$ (Gurevich, 1978). The magnetic field line which is mapped to the Troms $\varnothing$ site $(69.58 \mathrm{~N}$, 

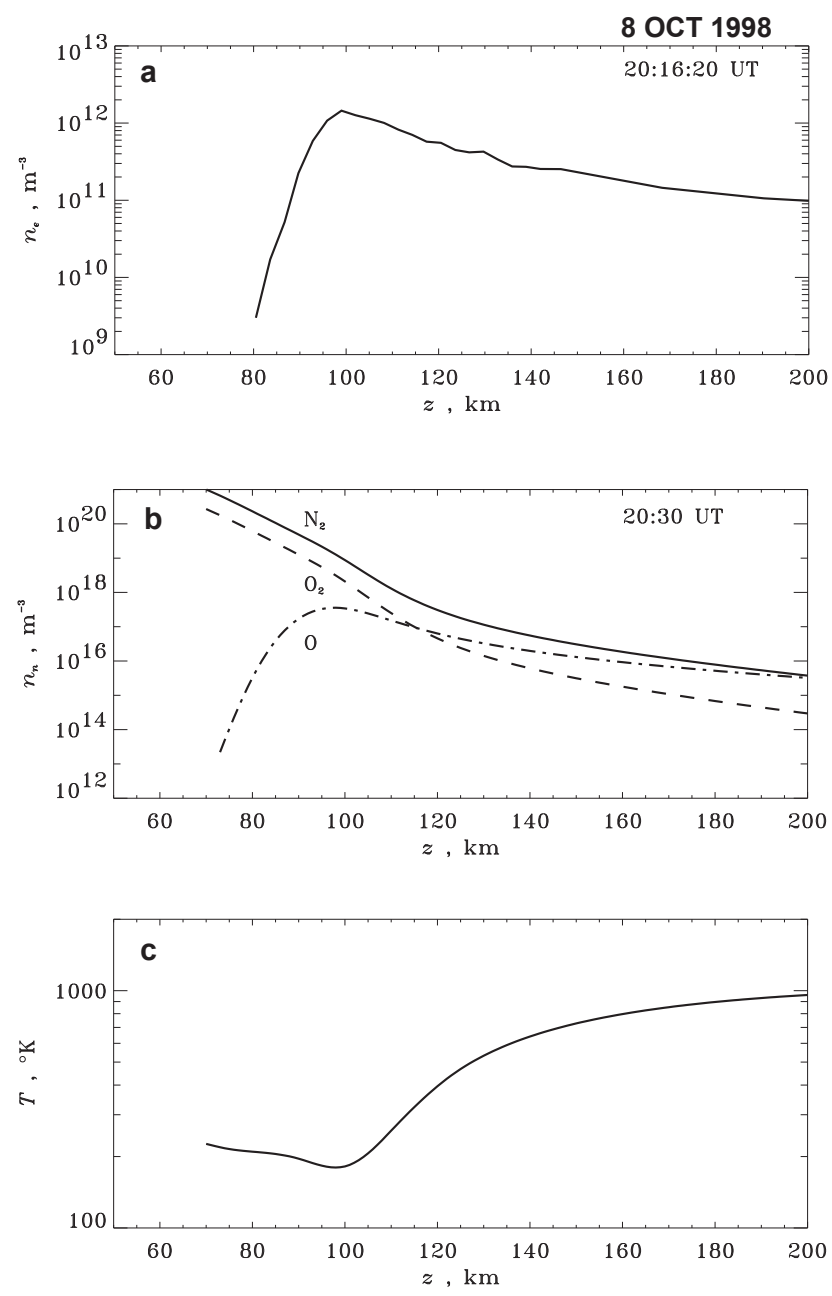

Fig. 2. Ionospheric conditions observed during the period of the FAST observations: (a) electron density $n_{e}$ measured by the EISCAT UHF incoherent scatter radar; (b) molecular composition $n_{n}$ and (c) temperature in the midnight sector as deduced from the ionospheric model MSIS90.

19.22 E geographic) may be approximated by a straight line up to $\sim 2.5 R_{E}$ and the magnetic strength at the geocentric distance $\rho$ along the magnetic field line is, to a good approximation, $\left|\boldsymbol{B}_{o}\right|=B_{o}\left(R_{E} / \rho\right)^{3}\left(1+3 \sin ^{2} \theta\right)^{1 / 2}$, where $R_{E}$ is the Earth's radius $\left(R_{E} \approx 6371 \mathrm{~km}\right), B_{o} \approx 3.1 \cdot 10^{4} \mathrm{nT}$ and $\theta$, the angle of magnetic latitude measured from the equator, is $\sim 58^{\circ}$. Electron-neutral and ion-neutral collision frequencies, electron plasma frequency and gyrofrequency, Pedersen and Hall conductivities which correspond to the ionospheric conditions in Fig. 2 are presented in Fig. 3.

\section{Model of polarization electric field and currents}

\subsection{Wave absorption}

The conditions of enhanced electron precipitation into the lower ionosphere indicated above lead to strong increases of both the absorption and refraction effects of the transmitted
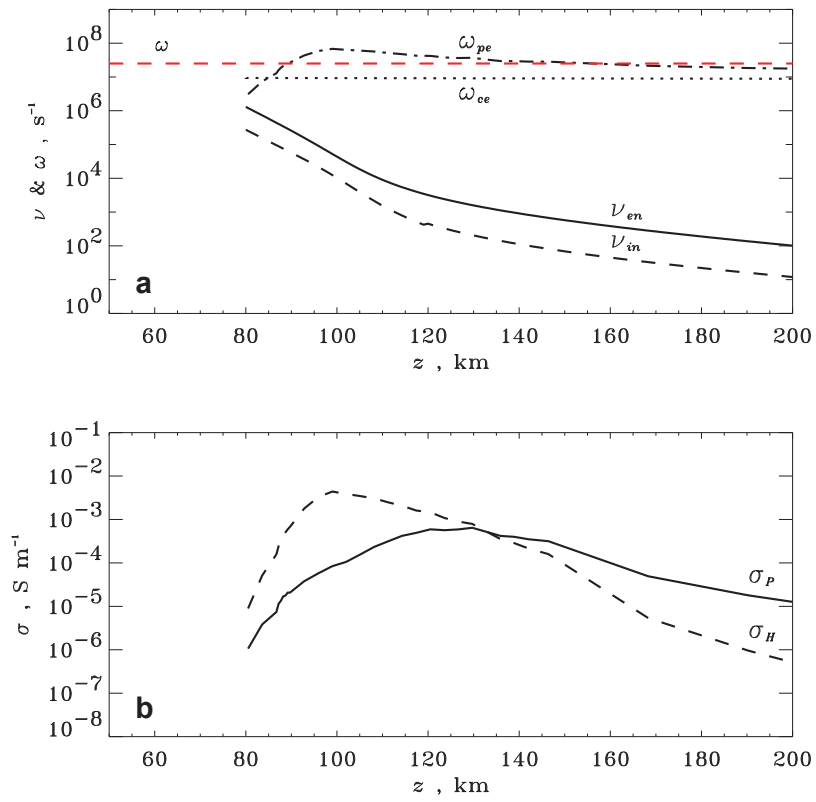

Fig. 3. (a) Electron-neutral (solid line) and ion-neutral collision frequencies (dashed line), electron plasma frequency (dashed-dotted line) and gyrofrequency (dotted line), HF pump frequency (dashed horizontal line), (b) the Pedersen (solid line) and the Hall (dashed line) conductivities which correspond to the ionospheric conditions of Fig. 2.

HF waves. The ordered energy emitted from the antenna is transformed into heat and electromagnetic noise mainly due to the electron collisions with neutral molecules and the wave amplitude decays quasi-exponentially with height as

$E_{\text {oK }}(z)=E_{o}(z) \cdot \exp \left(-\int_{z_{o}}^{z} \kappa d s\right)$,

where $E_{o}$ is defined by the Eq. (1), $z_{o}$ is the lower boundary of the ionosphere and $\kappa$ the absorption coefficient, which may be found from the Appleton formula for a complex refractive index $n$ of extraordinary $X$ wave. With the assumption that the $\boldsymbol{k}$ vector is aligned with the magnetic field line the refractive index may be written in the form (Davies, 1990)

$n^{2}=(\mu-i \chi)^{2}=M-i N$,

with $M=1-\omega_{p e}^{2}\left(\omega-\omega_{c e}\right) /\left\{\omega\left[\left(\omega-\omega_{c e}\right)^{2}+v_{e n}^{2}\right]\right\}$, $N=\omega_{p e}^{2} \nu_{e n} /\left\{\omega\left[\left(\omega-\omega_{c e}\right)^{2}+v_{e n}^{2}\right]\right\}$, where $\mu$ and $\chi$ are the real and the imaginary parts of the refractive index, respectively, $c$ is the free speed of electromagnetic wave $(c \approx$ $\left.3 \cdot 10^{8} \mathrm{~m} \mathrm{~s}^{-1}\right), v_{\text {en }}$ the electron-neutral collision frequency, $\omega$ is the angular frequency of the pump wave $\left(\omega \approx 2.5 \cdot 10^{7}\right.$ radians $\mathrm{s}^{-1}$ in our case), and $\omega_{p e}$ and $\omega_{c e}$ are the electron plasmafrequency and gyrofrequency, respectively. In plasma with $v_{e n} \ll\left|\omega-\omega_{c e}\right|$ the refractive index is almost entirely real and the condition of the $\mathrm{X}$ wave reflection reads

$\omega \approx\left(\omega_{c e}+\sqrt{\omega_{c e}^{2}+4 \omega_{p e}^{2}}\right) / 2$. 

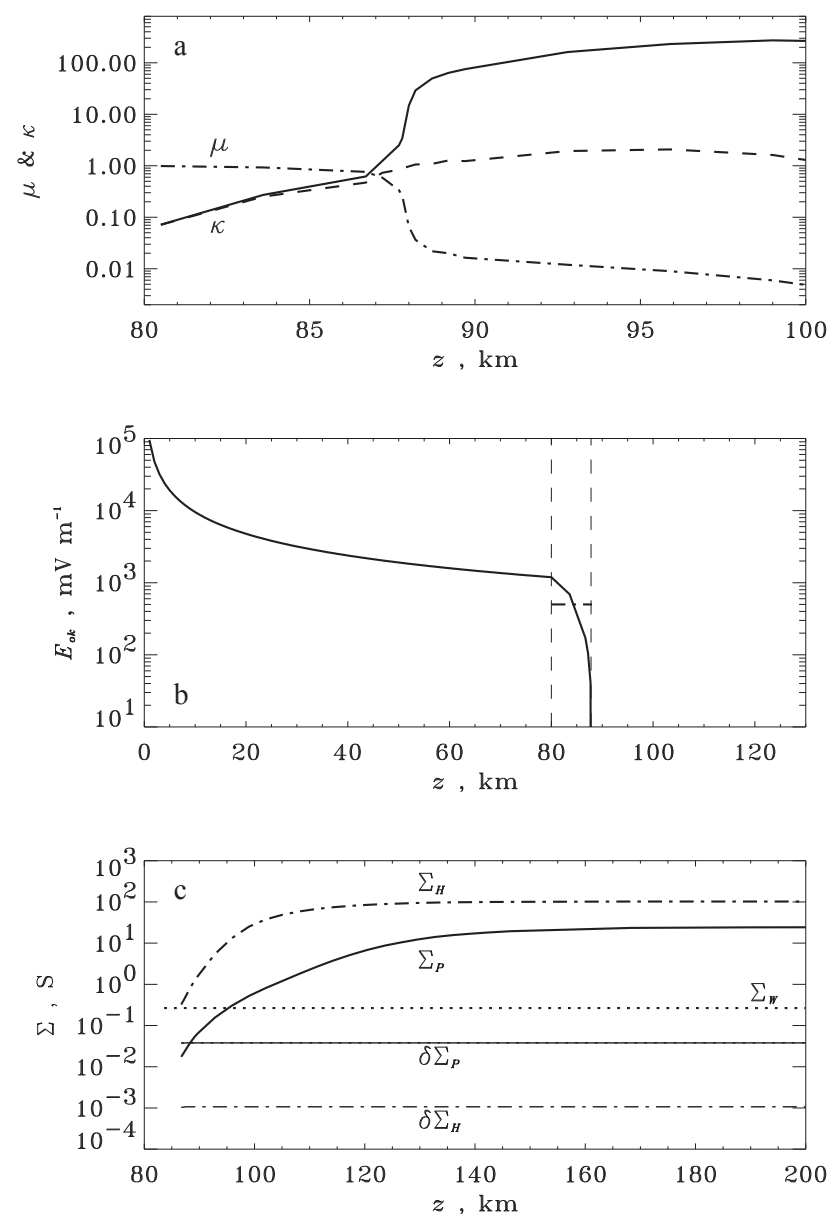

Fig. 4. (a) Variation with height of the real refractive index $\mu$ (dashed-dotted line) and the absorption coefficient $\kappa$ : the exact solution (6) is shown by a solid line and the approximated solution (5) by a dashed line. (b) Decay of the amplitude $E_{o k}$ of the transmitted HF wave with height. The absorption layer is limited by the vertical dashed lines. A characteristic plasma field $E_{p}$ in the absorption layer is shown by a horizontal dashed-dotted line. (c) Height integrated Hall, Pedersen, magnetospheric conductivities and their perturbations under the conditions discussed in Sects. 2.3, 3.1, 3.2.

When the real refractive index is close to unity the absorption coefficient may be approximately expressed as

$\kappa=\frac{\omega}{c} \frac{N}{2 \mu} \approx 0.53 \cdot 10^{-5} \frac{n_{e} v_{e n}}{\left[\left(\omega-\omega_{c e}\right)^{2}+v_{e n}^{2}\right]}$,

where the electron density $n_{e}$ is given in $\mathrm{m}^{-3}$ and $\kappa$ in neper $\mathrm{m}^{-1}$. Near the point of the wave reflection, approximation (5) is no longer valid and the general solution of the Eq. (3) has to be found. In general form $\kappa$ reads

$\kappa=\frac{\omega}{c} \frac{\sqrt{2}}{2} \sqrt{\sqrt{M^{2}+N^{2}}-M}$,

and the real refractive index is

$\mu=\frac{\sqrt{2}}{2} \sqrt{\sqrt{M^{2}+N^{2}}+M}$.
The variation with height of the absorption and refractive coefficients and the amplitude of electric field of the radiated HF $X$ wave are presented in Figs. 4a, b. The exact solution for the absorption coefficient (6), which is shown by a solid line in Fig. 4a, does not differ significantly from the approximate solution (5), given by a dashed line at the altitudes where the refractive index is close to unity. Near the reflection region where $\mu \rightarrow 0$ the two solutions differ significantly. The transmitted HF wave is absorbed in a thin layer between $\sim 80 \mathrm{~km}$, where the electron density commences to be significant, and the reflection point at $\sim 88 \mathrm{~km}$ (Fig. 4b). The absorbed part of the wave energy goes to the heating of the local plasma.

3.2 Modulation of the Pedersen and the Hall conductivities

Under the action of HF radio waves the electron temperature in the ionosphere may be increased considerably. The deviation of the electron temperature from the neutral temperature, $\delta T_{e}$, caused by the modulated wave consists of two parts: (i) a stationary perturbation

$\delta T_{e}^{(0)} \approx\left(1+\gamma^{2} / 2\right) T_{e o}\left[E_{o \kappa}(z) / E_{p}\right]^{2}$

and (ii) a modulated perturbation with frequency

$\Omega, \delta T_{e}^{(1)} \approx 2 \gamma T_{e o}\left[E_{o \kappa}(z) / E_{p}\right]^{2} ;$

here $T_{e o}\left(\right.$ in $K$ ) is the undisturbed temperature, $E_{p}$ is a characteristic plasma field which, in the case of the high frequency pump wave $\left(\omega \gg v_{e n}\right)$, is given by

$E_{p} \approx \sqrt{3 T_{e o} m_{e} e^{-2} \delta_{o} \omega^{2}}$

(Gurevich, 1978), where $\delta_{o}$ is the average fraction of the energy lost by the electron in one collision $\left(\delta_{o} \approx 10^{-3}\right)$. Therefore, if $E_{o \kappa} / E_{p} \sim 1$ (Fig. 4b) then the energy absorbed in the ionosphere will increase both the stationary and modulated parts of the electron temperature by a factor of $\sim 2$.

Due to the changes of electron temperature the electronneutral collision frequency and recombination coefficient are also modified. If we assume that the term $E_{\text {ок }} / E_{p} \leq 1$ then the collision frequency and recombination coefficient vary proportionally to the electron temperature, respectively as, $\delta v_{e n} / v_{e n} \approx 0.8 \delta T_{e} / T_{e o}$ and $\delta \alpha_{r} / \alpha_{r} \approx-0.7 \delta T_{e} / T_{e o}$ (Gurevich, 1978). Consequently the stationary part of plasma density follows the same law, namely $\delta n_{e} / n_{e} \approx 0.35 \delta T_{e} / T_{e}$. On the other hand, the modulated part does not exhibit any density change because the frequency of recombina-

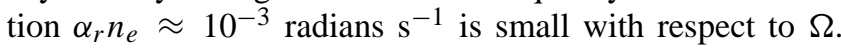
As a result, the perturbation of the modulated part of the Pedersen and the Hall conductivities in the plasma, with $1 \gg v_{e n} / \omega_{c e} \gg \omega_{c i} / v_{\text {in }}$ (which represents the ionospheric conditions below $\sim 90 \mathrm{~km}$ ), may be written

$\delta \sigma_{P} \approx 0.8 \frac{\delta T_{e}^{(1)}}{T_{e o}} \sigma_{p}$, 
$\delta \sigma_{H} \approx-1.6\left[\frac{\nu_{e n}}{\omega_{c e}}\right]^{2} \frac{\delta T_{e}^{(1)}}{T_{e o}} \sigma_{H}$,

with $\sigma_{P} \approx\left(e n_{e}^{\prime} / B\right)\left(v_{e n}^{\prime} / \omega_{c e}\right)$ and $\sigma_{H} \approx$ $e n_{e}^{\prime} / B$, where $v_{e n}^{\prime}=v_{e n}\left(1+0.8 \delta T_{e}^{(0)} / T_{e o}\right)$ and $n_{e}^{\prime}=n_{e}\left(1+0.35 \delta T_{e}^{(0)} / T_{e o}\right)$ are the modified collision frequency and electron density, respectively, caused by the stationary perturbation of temperature in the heating layer.

Enhancement of the electron temperature in the absorption layer gives rise to an increase of the absorption coefficient of approximately $n_{e}^{\prime} v_{e n}^{\prime} /\left(n_{e} v_{e n}\right)$ times, under the conditions $\left|\omega-\omega_{c e}\right| \gg v_{e n}$. Consequently, the electron temperature will be changed, but the secondary modification of the temperature will be $\sim e^{-n_{e}^{\prime} v_{e n}^{\prime} /\left(n_{e} v_{e n}\right)}$ times less than the primary one and therefore may be neglected under the condition $E_{\text {ок }} / E_{p} \sim 1$.

\subsection{Polarization field and currents}

Changes in the conductivities in the presence of a dc external convection electric field $\boldsymbol{E}$ lead to the primary current $\boldsymbol{j}_{o}=\delta \hat{\sigma} \cdot \boldsymbol{E}$ that acts only in the heated layer. Hereafter it is assumed that the external electric field $\boldsymbol{E}$ is approximately constant in the lower ionosphere and $\boldsymbol{E} \cdot \boldsymbol{B}_{o} \approx 0$. Then in cylindrical coordinates $(r, \varphi, z)$, the components of the external electric field are $E_{r}=E \cos \varphi, E_{\varphi}=-E \sin \varphi$, $E_{z}=0$ and the primary current is $j_{o}=\left\{\delta \sigma_{P} E_{r}+\right.$ $\left.\delta \sigma_{H} E_{\varphi},-\delta \sigma_{H} E_{r}+\delta \sigma_{P} E_{\varphi}, 0\right\} \cdot \exp \left(-r^{2} / a^{2}\right)$. Such a current gives rise to a polarization electric field $\delta \boldsymbol{E}$ in the ionosphere together with a secondary current $\boldsymbol{j}_{\perp}^{\prime}=\hat{\sigma} \cdot \delta \boldsymbol{E}$ and a parallel current $\boldsymbol{j}_{z}^{\prime}$ which close the primary one. If we assume that all time dependent perturbed quantities vary as $e^{i \Omega t}$, then the electric field perturbation can be written in the form, $\delta \boldsymbol{E}=-\boldsymbol{\nabla} \phi-i \Omega \boldsymbol{A}$, where $\phi$ and $\boldsymbol{A}$ are scalar and vector potentials. Then Poisson's equation under conditions of quasi-neutrality and Ampere's law in the quasi-static approximation, together with the gauge condition $\nabla_{\perp} \cdot \boldsymbol{A}=0$, read

$\frac{\partial}{\partial z} \sigma_{\|}\left(\frac{\partial \phi}{\partial z}+i \Omega \boldsymbol{A}_{z}\right)+\sigma_{P} \Delta_{\perp} \phi+i \Omega \sigma_{H}(\boldsymbol{\nabla} \times \boldsymbol{A})_{z}=\nabla \cdot \boldsymbol{j}_{o}$,

$\Delta_{\perp} \boldsymbol{A}_{z}-i \Omega \mu_{o} \sigma_{\|} \boldsymbol{A}_{z}-\mu_{o} \sigma_{\|} \frac{\partial \phi}{\partial z}=0$,

$\Delta \boldsymbol{A}_{\perp}-i \Omega \mu_{o} \hat{\sigma} \cdot \boldsymbol{A}_{\perp}-\mu_{o} \hat{\sigma} \nabla_{\perp} \phi-\nabla_{\perp} \frac{\partial \boldsymbol{A}_{z}}{\partial z}=-\mu_{o} \boldsymbol{j}_{\boldsymbol{o}}$

where $z$ is the displacement along the magnetic field (increasing with altitude), $\sigma_{\|}$is the longitudinal conductivity and the permeability $\mu_{o}=4 \pi \cdot 10^{-7} \mathrm{H} \mathrm{m}^{-1}$.

In the upper ionosphere Eqs. (10) and (11a) may be expressed in the form (Borisov and Stubbe, 1997)

$\frac{\partial^{2} \phi}{\partial z^{2}}-i \Omega \mu_{o} \sigma_{P} \phi=0$,

with the generalized Pedersen conductivity, which includes the ion inertia, $\sigma_{P}=\omega_{p i}^{2}\left(v_{i n}+i \Omega\right) /\left\{\omega_{c i}^{2}+v_{i n}^{2}\right\}$, where
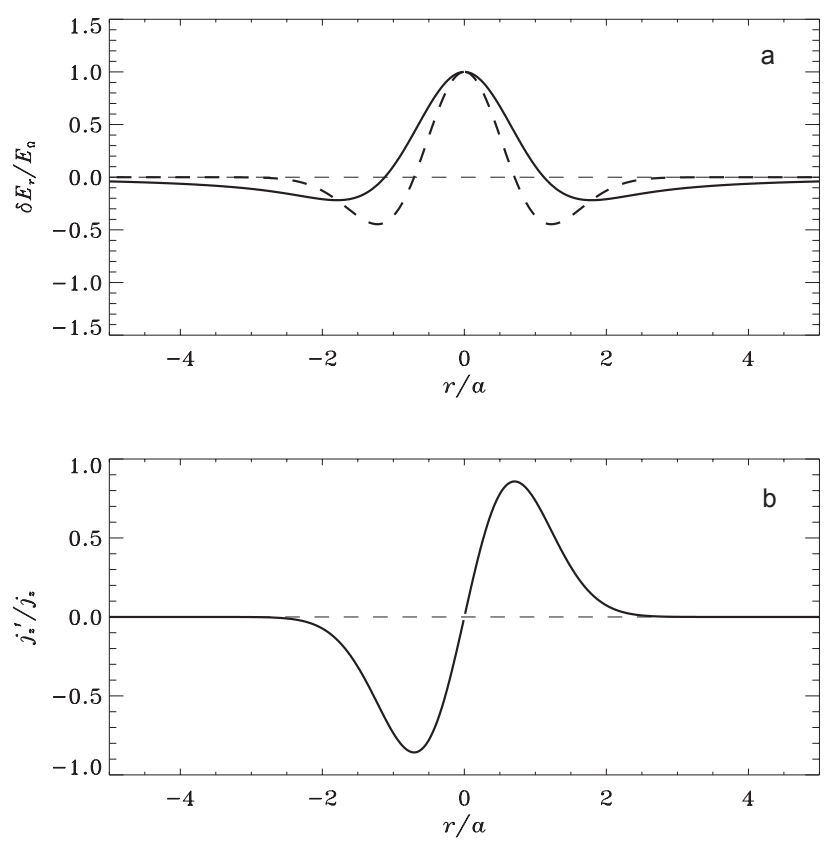

Fig. 5. (a) Normalised polarization electric field, $\delta E_{r} / E_{\Omega}$, in the case of weak skin effect (solid line) and $\left(k_{1} a\right)^{2} \delta E_{r} / E_{\Omega}$ under the conditions of strong skin effect (dashed line) versus normalised radial distance from the centre of heated spot; (b) radial profile of the parallel current $j_{z}^{\prime} / j_{z}$.

$v_{i n}$ is the ion-neutral collision frequency, $\sigma_{P}$ in $\mathrm{S} \mathrm{m}^{-1}$. At the heights where $\Omega>v_{i n}$, Eq. (12) describes the propagation of an Alfvén wave. In the lower ionosphere Eqs. (10), (11a, b), under the conditions $\mu_{o} \Omega \sigma_{P} \ll 1$, may be written (for the details see Borisov and Stubbe, 1997)

$$
\begin{gathered}
{\left[\frac{\partial}{r \partial r} r \frac{\partial}{\partial r}-\frac{1}{r^{2}}\right] \phi+\frac{i \Omega}{\Sigma_{W}+\Sigma_{P}} \int_{z_{o}}^{z_{i}} \sigma_{H} \Psi d z} \\
=\frac{1}{\Sigma_{W}+\Sigma_{P}} \int_{z_{o}}^{z_{i}} \nabla \cdot \boldsymbol{j}_{o} d z, \\
{\left[\frac{\partial^{2}}{\partial z^{2}}+\frac{\partial}{r \partial r} r \frac{\partial}{\partial r}-\frac{1}{r^{2}}\right] \Psi+\mu_{o} \sigma_{H}} \\
\cdot\left[\frac{\partial}{r \partial r} r \frac{\partial}{\partial r}-\frac{1}{r^{2}}\right] \phi=0,
\end{gathered}
$$

where $\Sigma_{P}$ and $\Sigma_{H}$ are the height integrated Pedersen and Hall conductivities; $\Sigma_{W}$ is the generalized magnetospheric conductance which takes the form $\Sigma_{W}=\left[1+i V_{A}\left(z_{i}\right) /(\Omega h)\right] /\left(\mu_{o} V_{A}\left(z_{i}\right)\right)$; the variation of the Alfvén speed with height is approximated by the polynomial

$V_{A}(z)=V_{A}\left(z_{i}\right)\left[1+\left(z-z_{i}\right) / h\right]^{2}$,

$z_{o}$ is the altitude which corresponds to the lower boundary of ionosphere, $z_{i}$ is the altitude which corresponds to the upper boundary of the ionosphere where $v_{i n} \sim \Omega$ and $h$ is the characteristic scale of the Alfvén speed variation. The solenoidal 
component is assumed to be dependent on the azimuthal angle $\varphi$, i.e., $(\boldsymbol{\nabla} \times \boldsymbol{A})_{z}=\Psi(r, z) \cdot \cos \left(\varphi+\varphi_{\Sigma}\right)$. Also, the right hand part of Eq. (13) can be simplified to

$\int \nabla \cdot \boldsymbol{j}_{\boldsymbol{o}} d z=2 \frac{r}{a^{2}} e^{-r^{2} / a^{2}} \delta \Sigma_{\Omega} E \cos \left(\varphi+\varphi_{\Sigma}\right)$,

with $\delta \Sigma_{\Omega}=\sqrt{\delta^{2} \Sigma_{P}+\delta^{2} \Sigma_{H}}, \varphi_{\Sigma}=\arctan \left(\delta \Sigma_{H} / \delta \Sigma_{P}\right)$, where $\delta \Sigma_{P}, \delta \Sigma_{H}$ are the height integrated perturbations of the Pedersen and Hall conductivities in the centre of the heated region. Making the Fourier-Bessel transform of Eqs. (13) and (14) with respect to $r$ and using definitions

$$
\begin{gathered}
\phi(r)=\int k J_{1}(k r) \phi(k) d k \text { and } \\
\Psi(r)=\int k J_{1}(k r) \Psi(k) d k,
\end{gathered}
$$

yields

$$
\begin{aligned}
-k^{2} \phi & +\frac{i \Omega}{\Sigma_{W}+\Sigma_{P}} \int_{z_{o}}^{z_{i}} \sigma_{H} \Psi d z \\
& =4 E_{\Omega} \int \frac{r^{2}}{a^{2}} e^{-r^{2} / a^{2}} J_{1}(k r) d r, \\
& {\left[\frac{\partial^{2}}{\partial z^{2}}-k^{2}\right] \Psi-\mu_{o} \sigma_{H} k^{2} \phi=0 . }
\end{aligned}
$$

Above, $E_{\Omega}=\delta \Sigma_{\Omega} E /\left\{2\left(\Sigma_{P}+\Sigma_{W}\right)\right\}$ and $J_{1}$ is the Bessel function of the first kind. The solution of (19) is

$$
\Psi=0.5 \mu_{o} k \phi \int \sigma_{H}(u) e^{-k|z-u|} d u
$$

and strongly depends on the profile of the Hall conductivity. Under the conditions $\Delta z \approx a$ this reduces to

$\Psi \approx 0.5 \mu_{o} k \phi \sigma_{o}^{-1} \int \sigma_{H}^{2} d u$,

where $\Delta z$ is a half-thickness of the Hall layer and $\sigma_{o}$ is the maximum value of the Hall conductivity. Substituting the solution for $\Psi$ to (18) and performing the inverse FourierBessel transform, yields

$\left[\frac{\partial}{r \partial r} r \frac{\partial}{\partial r}-\left(k_{1}^{2}+\frac{1}{r^{2}}\right)\right] \phi=4 E_{\Omega} \frac{r}{a^{2}} e^{-r^{2} / a^{2}}$,

with $k_{1}^{2} \approx\left(i \Omega \mu_{o} \pi / a\right) \Sigma_{H} \sigma_{o}^{-1} \int \sigma_{H}^{2} d z /\left\{2\left(\Sigma_{W}+\Sigma_{P}\right)\right\}$. The contribution of the solenoidal component in the polarisation electric field can be omitted if the condition

$\mu_{o} \Omega(a / \pi)^{2} \sigma_{H} \ll 1$

is satisfied. In this case, $k_{1} \approx 0$ and Eq. (22) has the solution

$\phi=-E_{\Omega} \frac{a^{2}}{r}\left(1-e^{-r^{2} / a^{2}}\right) \cos \left(\varphi+\varphi_{\Sigma}\right)$.

Therefore, the polarisation electric field is just

$$
\begin{aligned}
& \delta E_{r}=-E_{\Omega} \frac{a^{2}}{r^{2}}\left[1-\left(1+2 \frac{r^{2}}{a^{2}}\right) e^{-r^{2} / a^{2}}\right] \cos \left(\varphi+\varphi_{\Sigma}\right), \\
& \delta E_{\phi}=-E_{\Omega} \frac{a^{2}}{r^{2}}\left[1-e^{-r^{2} / a^{2}}\right] \sin \left(\varphi+\varphi_{\Sigma}\right)
\end{aligned}
$$

and the parallel current in the upper ionosphere is then simply calculated as

$$
\begin{aligned}
\dot{j}_{z}^{\prime}= & -\int_{z_{o}}^{z_{i}} \nabla \cdot\left(\boldsymbol{j}_{\boldsymbol{o}}+\boldsymbol{j}_{\perp}^{\prime}\right) d z \\
& =-2 j_{z} \frac{r}{a} e^{-r^{2} / a^{2}} \cos \left(\varphi+\varphi_{\Sigma}\right),
\end{aligned}
$$

with the magnitude of parallel current $j_{z}=\left(J_{o} / a\right)$ $\Sigma_{W} /\left(\Sigma_{W}+\Sigma_{P}\right)$ and the height integrated primary current $J_{o}=\delta \Sigma_{\Omega} E$. As a result, under conditions of weak skin effect, the magnitude of the parallel current depends on the matching between the Pedersen and magnetospheric conductancies. Further, if $\Sigma_{W} \ll \Sigma_{P}$ then the primary current is mainly closed by the perpendicular secondary current and only an insignificant fraction leaks into the magnetosphere along the field line. The variation of the normalised polarisation electric field $\delta E_{r} / E_{\Omega}$ and parallel current $\boldsymbol{j}_{z}^{\prime} / j_{z}$ versus normalised radial distance from the centre of the heated region are shown in the Fig. 5. Now let us consider the case when the Hall conductivity is large and the condition (23) is no longer satisfied. To make an analytical estimation, an approximate profile of the Hall conductivity of form, $\sigma_{H}(z)=\sigma_{o} e^{-\left(z-z_{H}\right)^{2} / a^{2}}$ is assumed and the solution of Eq. (22) reads

$$
\begin{aligned}
\phi=-2 & \frac{E_{\Omega}}{a^{2}}\left[K_{1}\left(k_{1} r\right) \int_{o}^{r} u^{2} I_{1}\left(k_{1} u\right) e^{-u^{2} / a^{2}} d u\right. \\
& \left.+I_{1}\left(k_{1} r\right) \int_{r}^{\infty} u^{2} K_{1}\left(k_{1} u\right) e^{-u^{2} / a^{2}} d u\right],
\end{aligned}
$$

where $z_{H}$ is the altitude where the Hall conductivity reaches its maximum; $I_{1}, K_{1}$ are the modified Bessel functions and $k_{1}^{2} \approx i \Omega \mu_{o}[\pi / 2]^{3 / 2} \Sigma_{H} \sigma_{o} /\left\{\Sigma_{P}+\Sigma_{W}\right\}$. Using properties of the Bessel functions, Eq. (28) may be simplified to

$\phi \approx-2 E_{\Omega} \frac{r}{\left(k_{1} a\right)^{2}} e^{-r^{2} / a^{2}} \cos \left(\varphi+\varphi_{\Sigma}\right)$ if $k_{1} r \ll 1$,

or

$\phi \approx-E_{\Omega}(i+1) \frac{r}{\left(k_{1} a\right)^{2}} e^{-r^{2} / a^{2}} \cos \left(\varphi+\varphi_{\Sigma}\right)$ if $k_{1} r \gg 1$

and

$\delta E_{r} \approx E_{\Omega}(i+1) \frac{e^{-r^{2} / a^{2}}}{\left(k_{1} a\right)^{2}}\left(1-2 \frac{r^{2}}{a^{2}}\right) \cos \left(\varphi+\varphi_{\Sigma}\right)$.

It is seen from (31) and Fig. 5a (dashed line) that the polarisation electric field at large distances from centre decreases exponentially, in contrast to (25). Its magnitude is $\sim\left(k_{1} a\right)^{2}$ times less than in the case of the purely potential field and is determined by the coupling between the Hall and the Pedersen conductances. Under the ionospheric conditions discussed in Sects. 2.3, 3.1, 3.2 and presented in the Figs. 2 to 4 , i.e., $\mu_{o} \Omega(a / \pi)^{2} \sigma_{H} \sim 1$ and $\sigma_{o} \approx 4 \cdot 10^{-3} \mathrm{~S} \mathrm{~m}^{-1}$, $\Sigma_{P} \approx 24 \mathrm{~S}, \Sigma_{H} \approx 100 \mathrm{~S}, \Sigma_{W} \approx 0.3 \mathrm{~S}, \delta \Sigma_{\Omega} \approx 0.04 \mathrm{~S}$, $E \approx 12 \mathrm{mV} \mathrm{m}^{-1}$, then $\left(k_{1} a\right)^{2} \approx 76.5$ and the primary current is closed mainly by the parallel current, the magnitude of which $j_{z} \approx J_{o} / a$, depends only on the height integrated 
primary current $\left(J_{o} \approx 0.4 \mathrm{~mA} \mathrm{~m}^{-1}\right.$ ) and the radius of heated spot and is estimated to be $j_{z} \approx 0.04 \mu \mathrm{A} \mathrm{m} \mathrm{m}^{-2}$.

The parallel current, which leaks into the magnetosphere, gives a perturbation of the transverse component of the magnetic field $B_{\perp} \approx\left(\mu_{o} / k_{\perp}\right) j_{z}^{\prime}$ and electric field $E_{\perp} \approx V_{A} B_{\perp}$. These perturbations propagate into the magnetosphere in the form of an Alfvén wave. For a sheared Alfvén wave which propagates in a plasma with the Alfvén speed profile given by polynomial (15) the variations of the transverse electric field with altitude are

$\left|E_{\perp}(z)\right| \approx\left|E_{\perp}\left(z_{i}\right)\right| \sqrt{V_{A}(z) / V_{A}\left(z_{i}\right)}$.

However, the profile with the polynomial law does not reflect the real variation of the Alfvén speed at the altitudes higher than $\sim 1000 \mathrm{~km}$, where the exponential law

$$
V_{A}(z)=V_{A}\left(z_{i}\right) e^{\left(z-z_{i}\right) / 2 h}
$$

is a more appropriate approximation. For the exponential profile the transverse electric field varies according to the Eq. (32) only in the low magnetosphere where $z \leq z_{i}+h$. At high altitudes it converges to a constant limit $\sim\left|E_{\perp}\left(z_{i}\right)\right| \sqrt{\pi h \Omega / V_{A}\left(z_{i}\right)}$ if the electron inertia length is small with respect to the transverse wavelength of the Alfvén wave. In the next section it will be demonstrated that for the transverse wavelength of $\sim 20 \mathrm{~km}$ the electron inertia determines the features of an Alfvén wave at altitudes of $\sim 2500$ $4000 \mathrm{~km}$.

\section{Properties of an Alfvén wave in plasma with exponen- tial law of Alfvén speed}

4.1 Electric and magnetic fields associated with Alfvén wave in inhomogeneous plasma

In the purely magnetohydrodynamic approximation Alfvén waves do not support an electric field with a component along $\boldsymbol{B}_{o}$. However, if the electron inertia is included the Alfvén wave does carry a parallel electric field in the plasma with low $\beta\left(\beta=2 \mu_{o} n_{e} k T / B^{2}\right)$. The relation between transverse and parallel electric fields of the wave is derived from Faraday's law

$$
\frac{\partial B_{\varphi}}{\partial t}=-\frac{\partial E_{r}}{\partial z}+\frac{\partial E_{z}}{\partial r},
$$

the parallel Ohm's law, neglecting the parallel electron pressure

$$
\frac{\partial j_{z}}{\partial t}=\frac{n_{e} e^{2}}{m_{e}} E_{z}
$$

and Ampere's law, neglecting the displacement current

$$
\frac{1}{r} \frac{\partial}{\partial r}\left(r B_{\varphi}\right)=\mu_{o} j_{z}
$$

Here $\partial / \partial \varphi=0$ is assumed. The combination of (34-36) yields

$$
\left(1-\lambda^{2} \frac{1}{r} \frac{\partial^{2}}{\partial r^{2}} r\right) E_{z}=-\lambda^{2} \frac{1}{r} \frac{\partial^{2}}{\partial r \partial z}\left(r E_{r}\right),
$$

with the electron inertial length $\lambda=c / \omega_{p e}$. If all parameters vary linearly as $\sim \exp \left(i \Omega t-i k_{z} z-i k_{r} r\right)$ with

$k_{r} \approx \pi / a(z)$,

and

$k_{z}=\Omega \sqrt{1+\lambda^{2} k_{r}^{2}} \sqrt{1+V_{A}^{2} / c^{2}} / V_{A}$,

then Eq. (37) may be simplified to

$\left(1+\lambda^{2} k_{r}^{2}\right) E_{z}=\lambda^{2} k_{r} k_{z} E_{r}$.

Note that to maintain constant magnetic flux along the flux tube, the perpendicular dimension of perturbation region must vary as $B_{o}^{-1 / 2}$ and therefore $\lambda^{2} k_{r}^{2} \sim B_{o} / n_{e}$. By this means, if an Alfvén wave propagates from the upper ionosphere $\left(z_{i} \sim 300 \mathrm{~km}\right)$ to $2550 \mathrm{~km}$ (altitude of FAST observations) the wavenumber $k_{r}$ decreases by approximately 1.5 times. If the dispersion relations (38), (39) are satisfied, then the parallel electric field grows in proportion to $\sim \Omega\left(n_{e} V_{A} a\right)^{-1} E_{r}$, in a plasma with $\lambda k_{r} \ll 1, V_{A} \ll c$ and in proportion to $\sim \sqrt{n_{e}} c \Omega^{-1} E_{r}$, when $\lambda k_{r} \gg 1, V_{A} \gg c$.

It is convenient to describe a sheared Alfvén wave in terms of a scalar potential $\phi$ and a vector potential $\boldsymbol{A}=A_{z} z$ where $\boldsymbol{B}=\nabla_{\perp} \times\left(A_{z} z\right)$. Substituting $E_{r}=i k_{r} \phi$ and $B_{\varphi}=i k_{r} A_{z}$ into (34-36) we obtain the following relation between $\phi$ and $A_{z}$,

$\frac{\partial \phi}{\partial z}=-i \Omega\left(1+k_{r}^{2} \lambda^{2}\right) A_{z}$.

Another relation between $\phi$ and $A_{z}$ comes from Ampere's law, including the displacement current (see, for example, Lysak, 1993), i.e.,

$\frac{\partial A_{z}}{\partial z}=-i \Omega \frac{1+V_{A}^{2} / c^{2}}{V_{A}^{2}} \phi$.

The combination of the last two equations gives

$\frac{\partial^{2} \phi}{\partial z^{2}}-\alpha_{1}(z) \frac{\partial \phi}{\partial z}+\alpha_{o}(z) \phi=0$,

with $\alpha_{1}(z)=2 k_{r}^{2} \lambda /\left\{1+k_{r}^{2} \lambda^{2}\right\} \quad \partial \lambda / \partial z$, $\alpha_{o}(z)=\Omega^{2}\left(1+k_{r}^{2} \lambda^{2}\right) /\left\{V_{A}^{2} /\left(1+V_{A}^{2} / c^{2}\right)\right\}$. By Faraday's and Ampere's laws, the perpendicular electric and magnetic fields must be continuous across the magnetosphereionosphere interface, and thus scalar and vector potentials must also be continuous at this boundary. The vector potential is driven by the parallel current which continuously leaks through the boundary. Then,

$A_{z}\left(z=z_{i}\right) \approx-\frac{i \mu_{o}}{k_{r}^{2}} j_{z}$.

The scalar potential is defined by the polarisation electric field, see Eq. (25) or (31). If the skin effect is strong, i.e., $k_{1} a \gg 1$, then $|\phi| \ll V_{A}\left(z_{i}\right)\left|A_{z}\right|$ and the boundary conditions are

$\phi\left(z=z_{i}\right) \approx 0$, 
and

$\frac{\partial \phi}{\partial z}\left(z=z_{i}\right) \approx-i \Omega A_{z} \approx \frac{\mu_{o} \Omega a^{2}}{\pi^{2}} j_{z}$

Equation (43) may be easily solved numerically (using a Runge-Kutta scheme) if the variation of coefficients $\alpha_{o}$ and $\alpha_{1}$ is known and the boundary conditions are defined.

The parallel electric field may be written as a function of scalar and vector potentials, as

$E_{z}=-i \Omega A_{z}-\frac{\partial \phi}{\partial z}=\left[\frac{1}{1+k_{r}^{2} \lambda^{2}}-1\right] \frac{\partial \phi}{\partial z}$.

If the electron inertial length is small, then the contribution of the vector potential cancels the contribution of scalar potential and the parallel electric field vanishes. When $\lambda$ grows, the contribution of vector potential to the parallel electric field decreases and for the conditions $k_{r} \lambda \gg 1$, the parallel electric field is entirely determined by the scalar potential.

In order to investigate the properties of the Alfvén wave a profile of Alfvén speed of the form (33) is chosen, with Alfvén speed in the upper ionosphere $V_{A}\left(z_{i} \approx 300 \mathrm{~km}\right) \approx 3 \cdot 10^{6} \mathrm{~m} \mathrm{~s}^{-1}$ and the characteristic height $h \approx 260 \mathrm{~km}$ which are found from fitting of the measured plasma density between 300 and $600 \mathrm{~km}$ by the EISCAT radar. The magnitude of parallel current in the upper ionosphere is taken as $j_{z} \approx 0.04 \mu \mathrm{A} \mathrm{m}^{-2}$, as estimated in the previous section. Variation with altitude of the electron density, the Alfvén speed, $\lambda k$ and $\lambda_{z}$, which are calculated according to the dispersion Eqs. (38), (39), are shown in the Fig. 6. The scalar potential, which is found from Eq. (43) with the boundary conditions (45), (46), is presented in Fig. 7a for two cases: (i) $\alpha_{1} \neq 0$, i.e. $\partial \lambda / \partial z \neq 0$ (solid line) and (ii) $\alpha_{1}=0$ (dashed line). It can been seen from the figure that from an altitude of $\sim 2300 \mathrm{~km}$, where the electron inertial length commences to be of the order of the transverse wavelength (i.e., $\lambda k_{r} \sim 1$ ), the two solutions diverge significantly. This means that from such altitudes inhomogeneous properties of the plasma play a major role. The conditions of Alfvén wave launching from the node of the potential $\left(\phi\left(z=z_{i}\right) \approx 0\right.$ at the boundary) are the most favourable to amplify the amplitude of wave at the highest altitudes. The amplitude of the potential will be proportional to the magnitude of the parallel current at the boundary. The node which appears at $\sim 1040 \mathrm{~km}$ above the upper ionospheric boundary indicates a partial reflection of the wave due to contribution of the gradient of Alfvén velocity to the refractive index and corresponds to the condition $V_{A}\left[\partial V_{A} / \partial z\right]^{-1}=2 h \approx \lambda_{z} / 2$. In the same figure the vector potential, multiplied by the Alfvén speed at the upper ionospheric boundary, $z=z_{i}$, is presented, again for the two conditions: (i) $\alpha_{1} \neq 0$ (dasheddotted line) and (ii) $\alpha_{1}=0$ (dotted line). Variations of transverse and parallel electric fields are shown in the Figs. 7b, c by solid lines. Here again the solution for the case $\alpha_{1}=0$ is presented by the dashed line. The amplitude of the transverse electric field at the altitude of the FAST observation ( $\sim 2550 \mathrm{~km}$ ) is found to be $\sim 1 \mathrm{mV} \mathrm{m}^{-1}$ (which is consistent
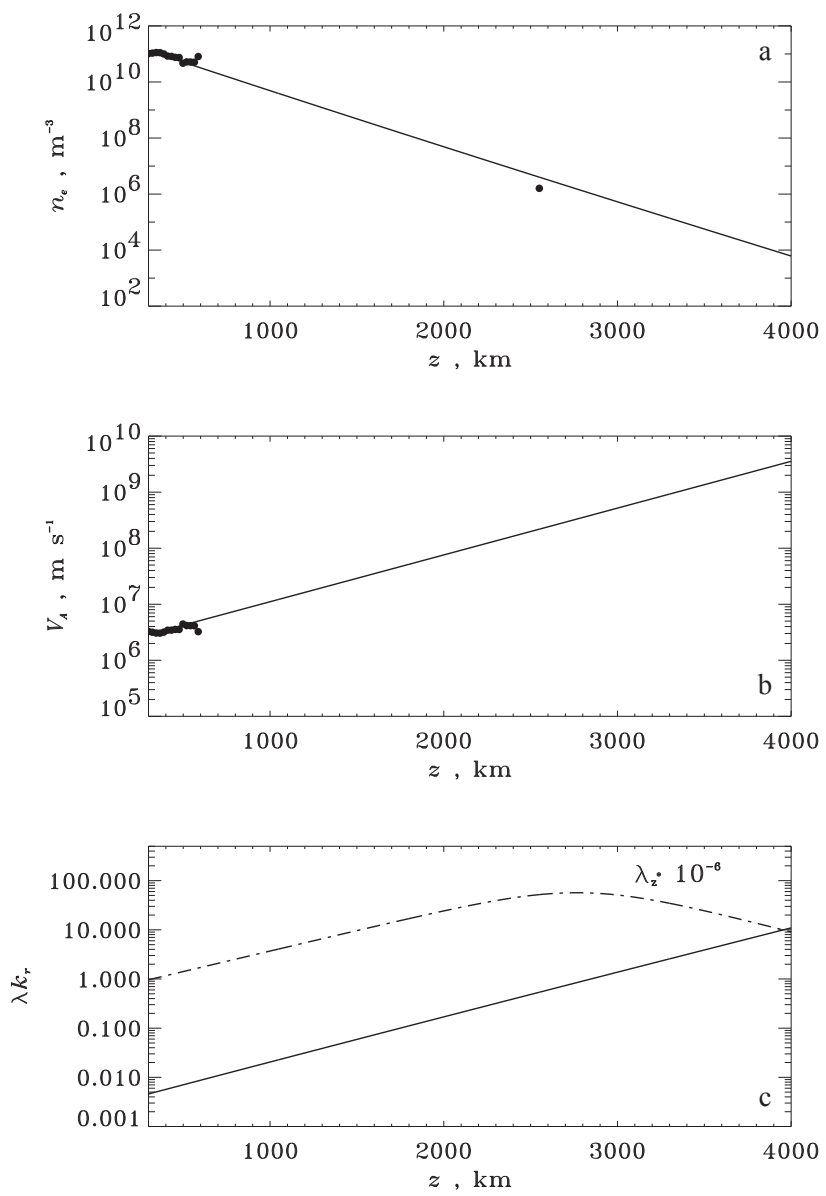

Fig. 6. Variation with altitude of (a) electron density $n_{e}$ : (i) which corresponds to the exponential profile of the Alfvén speed with $h=$ $260 \mathrm{~km}$ (solid line), (ii) measured by the EISCAT radar between 300 and $600 \mathrm{~km}$ (filled circles) and density of warm electron population which is estimated from the FAST observations at $2550 \mathrm{~km}$ (filled circle); (b) Alfvén speed given by an exponential law (solid line) with $h=260 \mathrm{~km}$, which fits the observational points between 300 and $600 \mathrm{~km}$ (filled circles); (c) longitudinal wavelength (dasheddotted line) and $\lambda k_{r}$ (solid line) as deduced from the dispersion Eqs. (38), (39).

with the FAST measurements) and parallel electric field to be $\sim 10^{-3} \mathrm{mV} \mathrm{m}^{-1}$ and increases exponentially with altitude.

Variations of transverse magnetic field and the parallel current (Fig. 8) deduced from the profile of the vector potential show that the expected amplitudes of these perturbations are small $\left(\sim 0.05 \mathrm{nT}\right.$ for magnetic field and $\sim 10 \mathrm{nA} \mathrm{m}^{-2}$ for the current) and lie near with the limit of the instrumental (magnetometer and particle detectors) resolution for the FAST spacecraft. 

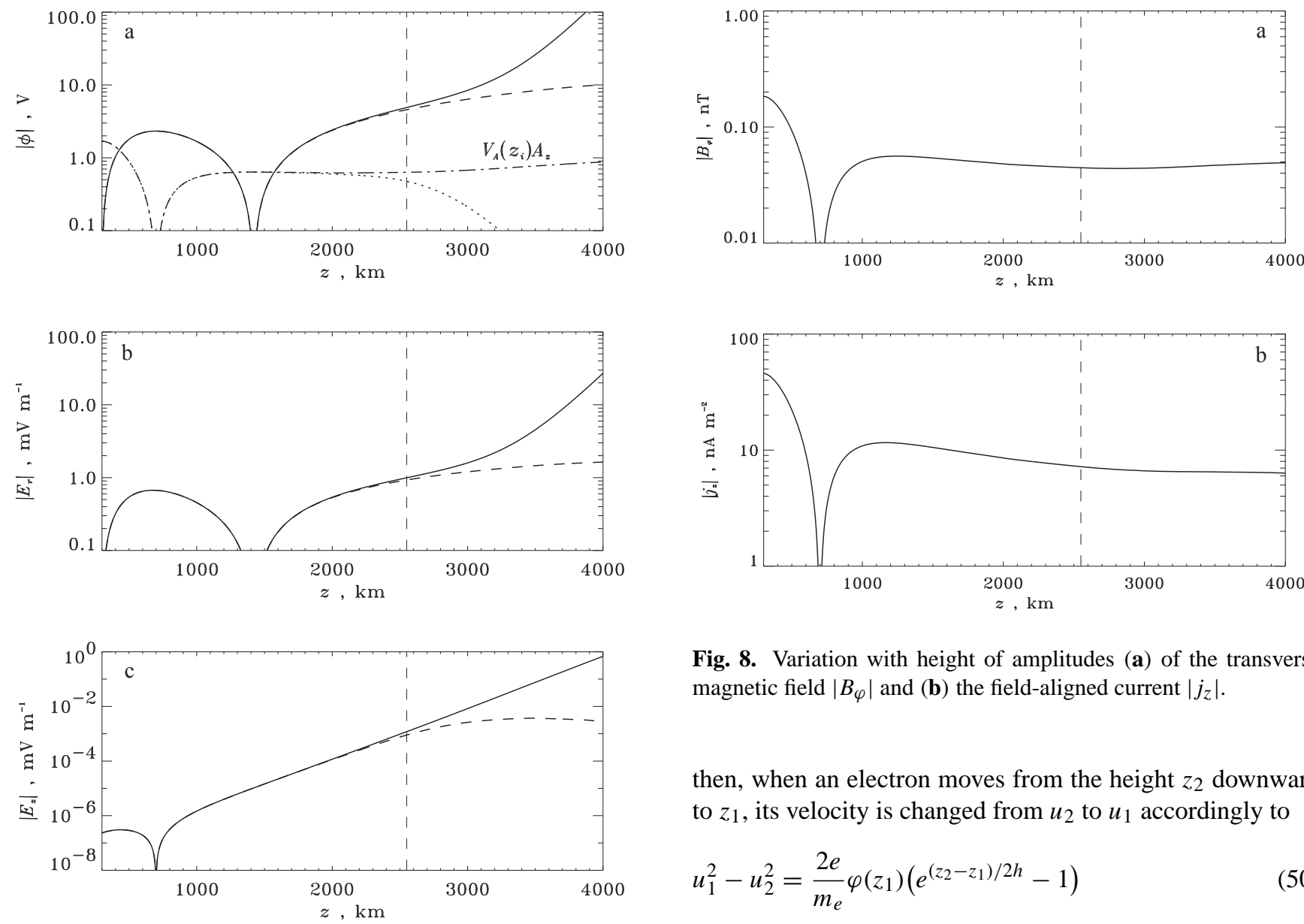

Fig. 8. Variation with height of amplitudes (a) of the transverse magnetic field $\left|B_{\varphi}\right|$ and (b) the field-aligned current $\left|j_{z}\right|$.

then, when an electron moves from the height $z_{2}$ downward to $z_{1}$, its velocity is changed from $u_{2}$ to $u_{1}$ accordingly to

$u_{1}^{2}-u_{2}^{2}=\frac{2 e}{m_{e}} \varphi\left(z_{1}\right)\left(e^{\left(z_{2}-z_{1}\right) / 2 h}-1\right)$

Fig. 7. Variation with height of (a) the scalar/vector potential shown for two cases: $\alpha_{1} \neq 0$ (solid/dashed-dotted line) and $\alpha_{1}=0$ (dashed/dotted line); (b) the amplitude of the transverse electric field $\left|E_{r}\right|: \alpha_{1} \neq 0$ (solid line), $\alpha_{1}=0$ (dashed line); (c) the parallel electric field $\left|E_{z}\right|: \alpha_{1} \neq 0$ (solid line), $\alpha_{1}=0$ (dashed line). Location of the FAST spacecraft is indicated by the vertical dashed line.

4.2 Electron acceleration by the parallel field of an Alfvén wave

Under the action of a parallel electric field, electrons will be accelerated according to

$\frac{\partial u}{\partial t}+u \frac{\partial u}{d z}=-\frac{e}{m_{e}} E_{z}$,

where $u$ is the field-aligned component of the electron velocity. If the parallel electric field is modulated with a period $\Delta t$, electrons will be accelerated downward (upward) during a half period and therefore the region of acceleration is limited. The upper boundary of the acceleration region may be estimated from simple considerations using the time delay between the electrons with different energies as observed by the FAST spacecraft. If we assume that the potential varies exponentially with altitude as

$\varphi(z)=\varphi\left(z_{1}\right) e^{\left(z-z_{1}\right) / 2 h}, z \geq z_{1}$,

and the flight time is estimated to be

$$
\begin{aligned}
t=\int_{z_{2}}^{z_{1}} & \frac{d z}{u}=4 h \int_{u_{2}}^{u_{1}} \frac{d u}{u^{2}-\left(u_{2}^{2}-\frac{2 e}{m_{e}} \varphi\left(z_{1}\right)\right)} \\
& =\frac{2 h}{\sqrt{u_{2}^{2}-\frac{2 e}{m_{e}} \varphi\left(z_{1}\right)}}\left[\operatorname { l n } \left(u+\sqrt{\left.u_{2}^{2}-\frac{2 e}{m_{e}} \varphi\left(z_{1}\right)\right)}\right.\right. \\
& \left.-\ln \left|u-\sqrt{u_{2}^{2}-\frac{2 e}{m_{e}} \varphi\left(z_{1}\right)}\right|\right]_{u=u_{1}}^{u=u_{2}} .
\end{aligned}
$$

The variations of the electron speed and the flight time with altitude are presented in Fig. 9 for two values of the electron velocity at the altitude $z_{1}=2550 \mathrm{~km}$, $u_{1}=3.6 \cdot 10^{6} \mathrm{~m} \mathrm{~s}^{-1}(37 \mathrm{eV})$ and $u_{1}=5.1 \cdot 10^{6} \mathrm{~m} \mathrm{~s}^{-1}(74 \mathrm{eV})$. Value of the potential at the altitude $z_{1}=2550 \mathrm{~km}$ and the characteristic spatial scale of the potential variation are taken from the results of the previous section, i.e., $\varphi\left(z_{1}\right)=5 \mathrm{~V}$ and $h=260 \mathrm{~km}$. Knowing the delay time $\sim 0.05 \mathrm{~s}$ between arrival of electrons with energies 37 and $74 \mathrm{eV}$, we conclude that the upper boundary of the acceleration region lies at $\sim 520 \mathrm{~km}$ above the spacecraft track.

Upward and downward electron fluxes measured by the electrostatic analyser in two energy channels with the centre energies 37 and $74 \mathrm{eV}$ (Fig. 1b, c) provide a possibility to reconstruct the distribution function. For simplicity we assume that a warm population may be reasonably described by an 

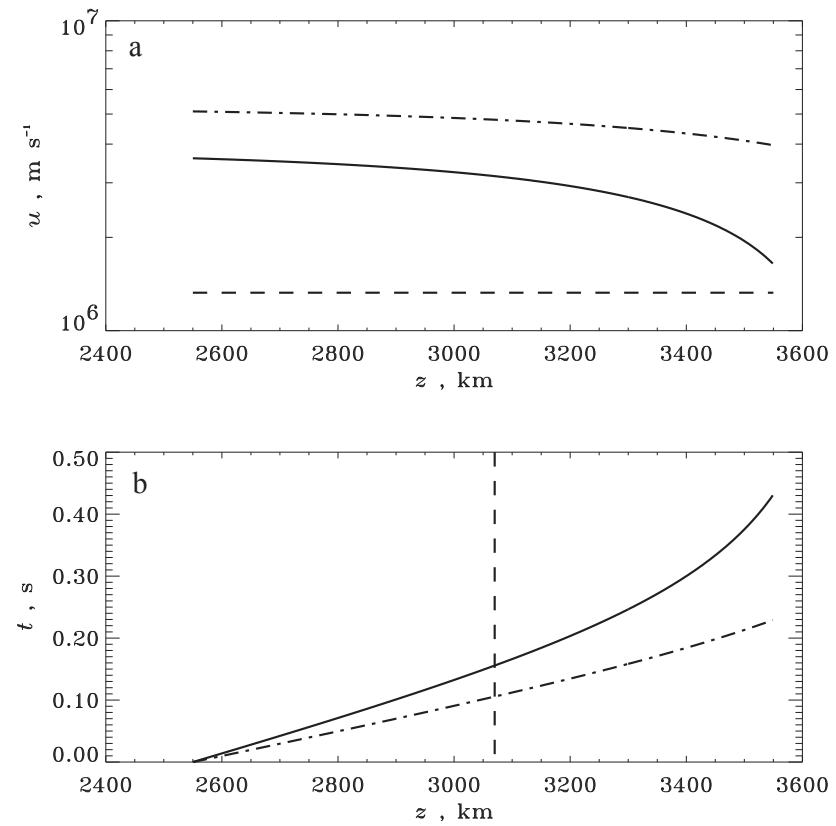

Fig. 9. Variation of (a) electron speed and (b) flight time with altitudes for two values of the electron velocity at the altitude $z_{1}=2550 \mathrm{~km}, 3.6 \cdot 10^{6} \mathrm{~m} \mathrm{~s}^{-1}$ (solid line) and $5.1 \cdot 10^{6} \mathrm{~m} \mathrm{~s}^{-1}$ (dashed-dotted line). The profile of the potential is taken in the form (49) with $\varphi\left(z_{1}\right)=5 \mathrm{~V}$ and $h=260 \mathrm{~km}$. A horizontal dashed line in the plot (a) presents the value of $\left(2 e / m_{e}\right) \varphi\left(z_{1}\right)$ and a vertical dashed line in the plot (b) shows the estimated upper boundary of the acceleration region.

isotropic Maxwell distribution with a bulk velocity along the field line, i.e.,

$f\left(n, \boldsymbol{v}_{\boldsymbol{o}}, T ; \boldsymbol{v}\right)=f_{o} \exp \left[-\frac{m\left[\boldsymbol{v}-\boldsymbol{v}_{\boldsymbol{o}}\right]^{2}}{2 T}\right]$,

which is a function of three parameters, density $n=$ $\pi^{3 / 2} f_{o}\left[2 T / m_{e}\right]^{3 / 2}$, bulk velocity $\boldsymbol{v}_{o}=\left\{0,0, u_{o}\right\}$ and temperature $T$. The electron flux $F$ recorded in a channel with centre energy $E$ is related to the distribution function as $F=\left(2 E / m_{e}^{2}\right) f$.

First, consider a "quiet" time period between 20:16:22 and 20:16:23 UT when the electron flux in each channel is approximately constant. Fitting four points (opened circles in Fig. 10) from the velocity space by the function (52) yields the following parameters: $n_{e}=1.6 \cdot 10^{6} \mathrm{~m}^{-3}$, $u_{o}=106 \mathrm{~km} \mathrm{~s}^{-1}, T=18.5 \mathrm{eV}\left(2.15 \cdot 10^{5} \mathrm{~K}\right.$ ) (solid line in the Fig. 10). Second, consider the observations around $\sim 20: 16: 21.6$ (Fig. 1b) when the particle detector which was pointed upward recorded a significant enhancement of the electron fluxes. The electron fluxes measured at that moment carry information about the distribution function of the warm population near with the upper boundary of the acceleration region. According to the Fig. 9a electrons with velocities $3.6 \cdot 10^{6} \mathrm{~m} \mathrm{~s}^{-1}$ and $5.1 \cdot 10^{6} \mathrm{~m} \mathrm{~s}^{-1}$ observed at the altitude $2550 \mathrm{~km}$ had the velocities $\sim 3.15 \cdot 10^{6} \mathrm{~m} \mathrm{~s}^{-1}$ and $\sim 4.8 \cdot 10^{6} \mathrm{~m} \mathrm{~s}^{-1}$, respectively, at the altitude $3070 \mathrm{~km}$.

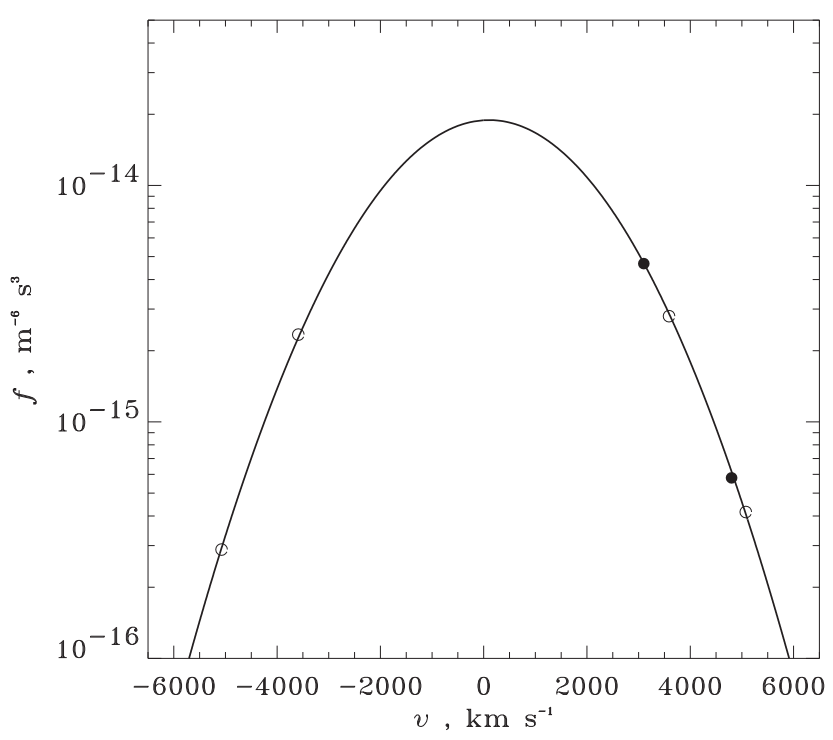

Fig. 10. Values of the background electron distribution function measured by the electrostatic analyser into the two energetic channels with central energies 37 and $74 \mathrm{eV}$ between 20:16:22 and 20:16:23 UT (open circles); together with the reconstructed distribution function of the warm electron population: (i) background population with $n=1.6 \cdot 10^{6} \mathrm{~m}^{-3}, u_{o}=106 \mathrm{~km} \mathrm{~s}^{-1}, T=18.5 \mathrm{eV}$ (solid line); (ii) values of the distribution function near the upper boundary of the acceleration region (filled circles).

Therefore the distribution function of the warm population near the upper boundary of the acceleration region may be reconstructed by making the corresponding shift of the measured distribution function in the velocity space. The resulting values of the distribution function, shown by the filled circles in the Fig. 10, demonstrate that parameters (density and temperature) of the warm population at the $530 \mathrm{~km}$ above the spacecraft track were approximately the same as observed locally.

Another important point that has to be mentioned here is the particle content of the magnetospheric flux tube. Generally speaking, a closed magnetic flux tube consists of two populations: a cold one of ionospheric origin and a warm one of magnetospheric origin. The exponential decrease of the plasma density with altitude is essentially due to the cold population. At the altitudes where the FAST observations were made and at least up to the upper boundary of the acceleration region the cold population has to be dominant to provide a continuous increase of Alfvén speed and consequently strong parallel electric field. Flattening of the density profile occurs at the altitudes where the densities of cold and superthermal populations are comparable and the density profile above these heights is defined by the warm population, which may be regarded as a constant in the first approximation. The altitude of the density flattening corresponds to the maximum of the Alfvén speed and presumably occurs near to the upper boundary of the acceleration region. 


\section{Conclusions}

In the present paper we propose a quantitative scenario of the Alfvén wave excitation by modulated HF heating of ionosphere. The Alfvén wave will be launched from the upper boundary of ionosphere whenever the following two conditions are simultaneously satisfied:

(i) a significant part of HF wave energy is absorbed in ionosphere;

(ii) there exists a dc electric field in the layer of absorption.

The mechanism of Alfvén wave excitation is as follows. The absorbed part of the ordered energy of the HF wave is transformed into the heating of the absorption layer, which leads to local perturbation of the conductivity with the modulated frequency. The dc electric field drives a primary current in the heated layer which is closed by polarisation current flowing in the ionosphere and by parallel current leaking into magnetosphere. Perturbations of electric and magnetic fields, which are associated with the parallel current, propagate into the magnetosphere in the form of an Alfvén wave. The parallel electric field of the Alfvén wave is significant in the region where the electron inertial length is of order of the transverse wavelength of the Alfvén wave or larger and may effectively accelerate superthermal electrons downward into the ionosphere.

The amplitude of the Alfvén wave is essentially determined by

(i) the magnitude of the primary current, which is bigger when the electron density and dc electric field in the absorption layer are larger;

(ii) the mechanism of closure of primary current, which depends on the matching between the ionospheric and magnetospheric conductances;

(iii) the amplitude of the parallel current, which is significant if the Hall conductance is large or if the Pedersen conductance is comparable with the magnetospheric conductance;

(iv) the conditions at the ionosphere-magnetosphere boundary;

(v) the Alfvén speed profile in magnetosphere.

Under the conditions of strong electron precipitation in the ionosphere, which took place during the FAST observations on 8 October 1998, the primary current caused by the perturbation of the conductivity in the heated region is closed entirely by the parallel current. In such circumstances the boundary conditions in the upper ionosphere are most favourable for the launching of an Alfvén wave: it is launched from the node in the potential gradient, which is proportional to the parallel current. Quantitative estimations obtained from this model are consistent with the FAST observations, i.e.,

(i) the amplitude of the transverse electric field is of the order of $\sim 1 \mathrm{mV} \mathrm{m}^{-1}$ compared to the $\sim 2-5 \mathrm{mV} \mathrm{m}^{-1}$ from the FAST measurements;

(ii) the parallel electric field with a magnitude of the order of $\sim 10^{-3} \mathrm{mV} \mathrm{m}^{-1}$ may effectively accelerate superthermal electrons downward into the ionosphere.

Acknowledgements. This work was supported by PPARC grant PPA/G/O/1999/00181. We thank N. Borisov (ISMIRAN) for useful discussions and R. Strangeway (University of California) for provision of the FAST data. We acknowledge the EISCAT staff for operating the facilities.

The Editor in Chief and authors thanks B. Lysak and M. Rietveld for their help in evaluating this paper.

\section{References}

Borisov, N. and Stubbe, P.: Excitation of longitudinal (fieldaligned) currents by modulated HF heating of the ionosphere, J. Atmos. Solar-Terr. Phys., 59, 1973-1989, 1997.

Davies, K.: Ionospheric Radio, IEE Electromagnetic waves series 31, Peter Peregrinus Ltd., 1990.

Gurevich, A. V.: Nonlinear phenomena in the ionosphere, (Ed) Roederer, J. G. and Wasson, J. T., Springer-Verlag, New York, 1978.

Hedin, A. E.: Extension of the MSIS thermosphere model into the middle and lower atmosphere, J. Geophys. Res., 96, 1159-1172, 1991.

Kimura, I., Stubbe, P., Rietveld, M. T., Barr, R., Ishida, K., Kasahara, Y., Yagitani, S., and Nagano, I.: Collaborative experiments by Akebono satellite, Troms $\varnothing$ ionospheric heater, and European incoherent scatter radar, Radio Sci., 29, 23-37, 1994.

Lyatsky, W., Belova, E. G., and Pashin, A. B.: Artificial magnetic pulsation generation by powerful ground-based transmitter, J. Atmos. Terr. Phys., 58, 407-414, 1996.

Lysak, R. L.: Generalized model of the ionospheric Alfvén resonator, in: Auroral Plasma Dynamics, (Ed) Lysak, R. L., 121128, AGU, Washington, 1993.

Pashin, A. B., Belova, E. G., and Lyatsky, W. B.: Magnetic pulsation generation by a powerful ground-based modulated HF radio transmitter, J. Atmos. Terr. Phys., 57, 245-252, 1995.

Robinson, T. R., Strangeway, R., Wright, D. M., Davies, J. A., Horne, R. B., Yeoman, T. K., Stocker, A. J., Lester, M., Rietveld, M. T., Mann, I. R., Carlson, C. W., and McFadden, J. P.: FAST observation of ULF waves injected into the magnetosphere by means of modulated RF heating of the auroral electrojet, Geophys. Res. Lett., 27, 3165-3168, 2000.

Stubbe, P. and Kopka, H.: Modulation of the polar electrojet by powerful HF waves, J. Geophys. Res., 82, 2319-2325, 1977. 